\title{
Structure Function Analysis of Mammalian Cryptochromes
}

\author{
F. Tamanini, I. Chaves, M.I. Bajek, AND G.T.J. van DeR Horst \\ Department of Genetics, Erasmus University Medical Center, 3000 CA Rotterdam, The Netherlands
}

\begin{abstract}
Members of the photolyase/cryptochrome family are flavoproteins that share an extraordinary conserved core structure (photolyase homology region, PHR), but the presence of a carboxy-terminal extension is limited to the cryptochromes. Photolyases are DNA-repair enzymes that remove UV-light-induced lesions. Cryptochromes of plants and Drosophila act as circadian photoreceptors, involved in light entrainment of the biological clock. Using knockout mouse models, mammalian cryptochromes (mCRY1 and mCRY2) were identified as essential components of the clock machinery. Within the mammalian transcription-translation feedback loop generating rhythmic gene expression, mCRYs potently inhibit the transcription activity of the CLOCK/BMAL1 heterodimer and protect mPER2 from 26S-protesome-mediated degradation. By analyzing a set of mutant mCRY1 proteins and photolyase/mCRY1 chimeric proteins, we found that the carboxyl terminus has a determinant role in mCRY1 function by harboring distinguished domains involved in nuclear import and interactions with other clock proteins. Moreover, the carboxyl terminus must cross-talk with the PHR to establish full transcription repression capacity in mCRY1. We propose that the presence of the carboxyl terminus in cryptochromes, which varies in sequence composition among mammalian, Drosophila, and plant CRYs, is critical for their different functions and possibly contributed to shape the different architecture and biochemistry of the clock machineries in these organisms.
\end{abstract}

\section{INTRODUCTION: THE PHOTOLYASE/ CRYPTOCHROME PROTEIN FAMILY}

Light/dark cycles, accompanied by the daily rise and fall in temperature, are well-known examples of environmental changes imposed by the rotation of the Earth around its axis. To anticipate these day/night cycles, most organisms have developed a self-sustained circadian clock that drives rhythmic changes in metabolism, physiology, and behavior. In mammals, the circadian system is composed of a master clock in the suprachiasmatic nuclei (SCN) of the hypothalamus in the brain and slave oscillators in peripheral tissues (Ralph et al. 1990; Reppert and Weaver 2002). Because the periodicity of the circadian pacemaker is not exactly 24 hours, it must be reset every day by environmental stimuli, among which light is the most predominant. Within the animal circadian system, cryptochromes hold a remarkable position because they have been shown to act either as integral components of transcription-translation feedback loops that make up the molecular oscillator or as light receptors required for photoentrainment of the circadian clock (Cashmore et al. 1999). Intriguingly, cryptochromes also show strong sequence and structural homology with photolyases, DNA-repair enzymes that remove UV-light-induced DNA lesions with the aid of visible light (Sancar 2003). Together, these proteins make up the photolyase/cryptochrome protein family of flavoproteins that share a highly homologous central core domain with two chromophores (photolyase homology region, PHR) and contain unique amino- and carboxy-terminal extensions, the latter likely providing the basis for totally distinct functions: (1) DNA repair (eukaryotic photolyases), (2) circadian photoentrainment (plant, zebra fish, and Drosophila cryptochromes), and (3) circadian clock gene expression (mammalian cryptochromes). Whereas the protein structure and reaction mechanism of photolyases have been largely resolved, relatively little such information is known about cryptochromes. Here, we summarize the current knowledge on the structure and function of cryptochromes, with emphasis on mammalian cryptochromes.

\section{PHOTOLYASES AND DNA REPAIR}

The light of our sun is essential for life on Earth. For example, visible light is mandatory for oxygen production by plants via photosynthesis. In mammals and other organisms, even the highly energetic UV component of sunlight is required for the synthesis of important substances such as vitamin D. In marked contrast to its beneficial effects, UV light causes DNA lesions that bring about cell death or, upon replication of a genome, cause somatic mutations (Friedberg 1996). Given the high levels of UV irradiation to which life forms in the Archeal world were exposed, it is not surprising that one of the first DNA-repair systems emerging during evolution, photoreactivation, focused on repair of UV-induced DNA damage.

Photoreactivation is a light-dependent enzymatic reaction in which photolyases directly revert cyclobutane pyrimidine dimers (CPDs) and pyrimidine 6-4 pyrimidone photoproduct (6-4PP) lesions into normal DNA bases. Photolyases are monomeric, single-chain proteins with a molecular mass of $50-65 \mathrm{kD}$, showing substrate specificity for either CPD or 6-4PP photolesions (Carell et al. 2001). The repair reaction starts with the light-independent binding of a single photolyase molecule to the DNA lesion, resulting in the formation of an enzyme-substrate complex. Next, the DNA dimer is split in a reaction requiring energy provided by (blue) light, after which the photolyase dissociates from the repaired DNA. Binding of photolyase to the DNA does not require the presence of light. Photolyases have been purified from a variety of species, and detailed information on the reaction mecha- 
nism is available (Tamada et al. 1997; Sancar 2003). Characteristic for photolyases is the presence of two different noncovalently bound chromophoric cofactors. The first chromophore, FAD, acts as a catalytic cofactor and is only biologically active in its fully reduced state. The latter is accomplished via a reaction called photoactivation, involving a unique feature of intraprotein electron transfer along a chain of three tryptophans over a distance of more than $13 \AA$, allowing the transfer of an electron to FAD (Aubert et al. 2000). The second chromophore, either a reduced folate or an 8-hydroxy-5-deazaflavin, serves as an auxillary light-harvesting antenna that transmits light energy to the FAD chromophore. Dimer splitting during photoreactivation occurs through electron donation. In the absence of the second chromophore, photolyase enzymes can still photosplit dimers, although the efficiency of the repair reaction is reduced. Comparison of photolyases revealed a strong conservation of amino acids involved in intraprotein electron transfer (during photoactivation) and FAD binding, as well as positively charged amino acids situated at the surface and potentially involved in DNA binding (Mees et al. 2004).

Although photolyases have been observed from bacteria and yeasts to plants and animals (including marsupials), the enzyme has not been identified in placental mammals and was apparently lost in evolution. Instead, mammals remove UV-induced CPDs and 6-4PPs (as well as other bulky strand-distorting adducts) by another evolutionary conserved repair mechanism, nucleotide excision repair (NER) (de Laat et al. 1999). Whereas 6-4PPs are rapidly repaired by NER, removal of CPDs is usually slow (for review, see Bohr et al. 1985). Despite their absence in placental mammals, we have shown that photolyases are functional in mice. Transgenic animals expressing the Arabidopsis thaliana (6-4PP)-photolyase and/or the Potorous tridactylis (rat-kangaroo) CPD-photolyase from the ubiquitous $\beta$-actin or keratinocyte-specific K14 promoter are viable and are capable of lesion-specifically repairing UV-induced 6-4PPs or CPDs in a light-dependent manner from the skin (Schul et al. 2002). We have shown that photoreactivation of CPDs in basal keratinocytes of UV-exposed animals dramatically reduced sunburn, mutation induction, and skin cancer formation (see Fig. 1). In marked contrast, photoreactivation of 6-4PPs hardly exerted any effect, which is likely due to the fact that NER repairs CPDs much more slowly than 64PPs (Garinis et al. 2005; Jans et al. 2005, 2006). Thus, these transgenic mice serve as tools to discriminate among the deleterious effects of each individual type of DNA damage and to understand their relative impact on skin cancer development, which is one of the most frequent causes of tumors in western society.

\section{CRYPTOCHROMES AND PHOTOTRANSDUCTION}

Not only is light used by organisms as an energy source (e.g., photosynthesis and photoreactivation), but it also allows them to perceive and respond to their environment. Evidently, this requires the presence of photoreceptor proteins that transfer light information to the organism.

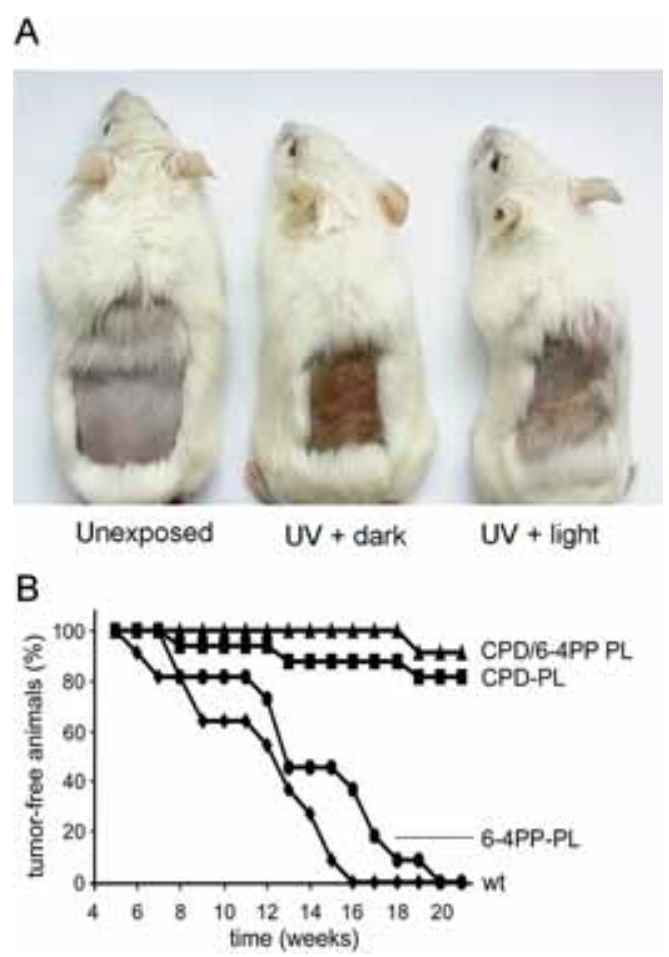

Figure 1. Effect of CPD photoreactivation on UV-induced erythema and carcinogenesis in the skin of $\beta$-act-CPD photolyase transgenic mice. (A) Appearance of the dorsal skin of non-UVexposed (left), UV-exposed (middle), and UV-exposed/photoreactivated (right) animals, showing clear erythema when photoreactivation of UV-exposed animals is omitted. $(B)$ Fraction of tumor-bearing wild-type and $\beta$-act-CPD photolyase (and $\beta$-act6-4PP) transgenic mice in time after the first UV/photoreactivation treatment. ( $A$, Reprinted, with permission, from Schul et al. 2002 [European Molecular Biology Organization]; $B$, reprinted, with permission, from Jans et al. 2005 [C Elsevier].)

Among such photoreceptor proteins are cryptochromes, initially discovered as plant blue light receptors (Cashmore 2003).

Arabidopsis cryptochrome 1 and 2 (AtCRY1 and AtCRY2) are nuclear proteins, resembling photolyases in having a central core domain (PHR) that binds two chromophores. Although lacking the nuclear and mitochondrial localization signal containing an amino-terminal extension that discriminates eukaryotic photolyases from their prokaryotic counterparts, they contain a unique carboxyterminal extension with different length and amino acid composition. AtCRY proteins were first identified as blue light photoreceptors, involved in light-induced stomatal opening, anthocyanin production, flowering, and inhibition of hypocotyl elongations (Suarez-Lopez and Coupland 1998; Cashmore et al. 1999).

AtCRY proteins detect light signals via the PHR, resulting in activation of their carboxy-terminal domain (Sang et al. 2005). This allows the protein to directly interact with and inhibit the COP1 (constitutive photomorphogenic 1) protein, which serves as a critical component for activation of the photomorphogenic gene expression program in plants (Wang et al. 2001). Interestingly, overexpression of the carboxy-terminal tails of AtCRY1 and AtCRY2 is suf- 
ficient to cause constitutive CRY activity (e.g., repression of COP1) (Yang et al. 2000). Therefore, in plants, it seems that the PHR of AtCRYs is mainly a regulator of the carboxyl terminus, whereas the latter domain exerts major function in phototransduction. Furthermore, light activation of photoreceptor activity requires homodimerization of AtCRY proteins via amino-terminal domains in the PHR (Sang et al. 2005). Little is known about the mechanism of light activation of AtCRY, although (similar to photolyases) the activity of the protein depends on the redox status of the FAD chromophore (Bouly et al. 2007).

The occurrence of cryptochromes is not limited to plants. Emery et al. (1998) isolated a fly with a missense mutation at a highly conserved flavin-binding residue of Drosophila CRY (dCRY). Circadian experiments with this mutant strain $\left(c r y^{\mathrm{b}}\right)$ revealed that when kept in constant darkness, flies no longer responded to brief light pulses that induce phase shifts (Stanewsky et al. 1998). In contrast, flies overexpressing a wild-type cry gene appeared to be hypersensitive to light-induced phase shifting. These data show that Drosophila CRY acts as a circadian photoreceptor for resetting the biological clock. Upon light activation, the dCRY protein directly interacts with clock proteins TIM and PER and causes proteasomal degradation of the protein complexes. In contrast, overexpression of a truncated dCRY protein, lacking the carboxyl terminus, causes constitutive CRY activity (Busza et al. 2004). Thus, in contrast to AtCRY, the carboxy-terminal domain of dCRY has a regulatory function, by preventing dCRY from being active in the dark.

\section{CRYPTOCHROMES AND THE CIRCADIAN CORE CLOCK}

In search of mammalian homologs of photolyases, we and other investigators have cloned two photolyase-like genes. Because the gene products do not possess photolyase activity and the PHRs lack the amino-terminal extension of eukaryotic photolyases and instead contain a carboxy-terminal extension also present in plant cryptochromes, the genes were designated $m C r y 1$ and $m$ Cry 2 (van der Spek et al. 1996; Todo et al. 1997).

Studies with genetically modified mice in which the $m C r y 1$ and/or $m C r y 2$ genes were inactivated revealed that the two mouse cryptochromes are part of the molecular clockwork generating behavioral and molecular rhythms. Notably, single-knockout mice have opposing circadian phenotypes, as evident from the observation that $m C r y 1^{-/-}$ and $m C r y 2^{-/-}$mice display short and long behavioral periodicity, respectively, as measured by voluntary wheel-running activity (van der Horst et al. 1999; Vitaterna et al. 1999). Remarkably, behavioral analysis of $m C r y 1 / 2$ double-knockout mice indicated a complete loss of the circadian clock in these animals (van der Horst et al. 1999). Thus, mCRY1 and mCRY2 proteins not only have an antagonistic clock-adjusting function, but are also essential for maintenance of circadian rhythmicity.

Unexpectedly, given the anticipated photoreceptor function predicted on the basis of the homology with plant cryptochromes, $m C r y 1 / 2$ double-knockout mice still show inhibition of wheel-running activity by light and induction of mPer 1 and $m P e r 2$ expression upon exposure to light pulses known to reset the biological clock, suggesting that mCRY1 and mCRY2 are not required for photoentrainment or light masking of activity (Okamura et al. 1999).

How do CRY proteins fit in the circadian clockwork? Until the discovery of animal cryptochromes, the mammalian molecular oscillator was thought to be composed of mPer 1, mPer2, and mPer3 genes (homologs of the Drosophila period gene) and clock and Bmall genes. In the transcription-translation feedback loop model, transcription of mPer genes is driven by a heterodimer of the CLOCK and BMAL proteins (positive loop) that binds to CACGTG E-box enhancer elements in the promoter of Per genes (Ripperger et al. 2000). Following translation of mPer mRNAs, mPER proteins were thought to inhibit CLOCK/BMAL1 and, accordingly, switch down transcription of their own genes (negative loop). After mPER protein levels are down again, transcription resumes and the next cycle can start. In line with their clock function, as suggested by animal experiments, the $m$ Cry 1 and $m$ Cry 2 genes are expressed in a circadian manner, whereas simultaneous inactivation of the $m C r y$ genes abolishes cycling of mPer 1 and $m P e r 2$ expression (Okamura et al. 1999). A key observation in disclosing the function of cryptochrome genes was the finding that CRY proteins are much stronger inhibitors of CLOCK/BMAL1-driven transcription of E-box-containing reporter genes than mPER proteins (Kume et al. 1999). This observation placed CRY proteins unequivocally at the core of the circadian oscillator and pointed to them as being the most important factors in maintaining the negative feedback loop of the molecular oscillator, despite the fact that other proteins, such as DEC1 and DEC2, have also been proposed to contribute (Honma et al. 2002).

Interestingly, the function of mCRY1 and mCRY2 in the molecular oscillator is not exclusively confined to the level of transcription repression; the proteins also have a role in posttranslational control of other core clock proteins. Coimmunoprecipitation studies with transiently expressed clock proteins and yeast two-hybrid experiments have uncovered direct interactions between mCRY proteins and various other core oscillator components (i.e., mPER2, mPER1, CLOCK, and BMAL1) (Shearman et al. 2000; Yagita et al. 2002; Chaves et al. 2006). In addition, immunohistochemical and biochemical studies revealed synchronous circadian patterns of abundance, phosphorylation status, and nuclear localization of mCRY and mPER proteins (Lee et al. 2001). Particularly, the observation that $m P e r 2$ mRNA levels are increased in $m C r y 1 / 2$ double-knockout mice, whereas mPER 2 protein could not be detected in the cytoplasm or the nucleus of SCN neurons (Shearman et al. 2000), suggested that mCRY proteins are necessary to stabilize mPER2. Indeed, physical interaction between mPER2 and mCRY proteins was shown to inhibit PER2 ubiquitylation and proteasomal degradation (and possibly changes in subcellular localization) of the mCRY-mPER complex (Yagita et al. 2002). Thus far, known protein-protein interactions of, as well as CLOCK/BMAL1-mediated transcription inhibition by, mCRY proteins have been shown to be independent from light (Griffin et al. 1999). 
Unexpectedly, given the anticipated photoreceptor function predicted on the basis of homology with plant cryptochromes, $m$ Cry $1 / 2$ double-knockout mice still show inhibition of wheel-running activity by light and induction of $m P e r 1$ and $m P e r 2$ expression upon exposure to light pulses known to reset the biological clock, suggesting that $\mathrm{mCRY} 1$ and $\mathrm{mCRY} 2$ are not required for photoentrainment or light masking of activity (Okamura et al. 1999). As such, mCRY proteins differ from dCRY proteins, which, in addition to functioning as a circadian photoreceptor, had been shown to be involved in core clock oscillations in peripheral tissues (Emery et al. 1998; Stanewsky et al. 1998).

\section{STRUCTURE/FUNCTION ANALYSIS OF MAMMALIAN CRYPTOCHROMES}

We have chosen to perform a detailed structure/function analysis of cryptochromes to (1) obtain mechanistic insight into the mode of action of CRY1 and CRY2 proteins in the circadian core oscillator, with special emphasis on the function of the carboxy-terminal extension, (2) determine whether photoreactivation and clock functions are mutually exclusive or whether it is possible to combine them in the same protein, and (3) determine how nature can use the same core sequence for completely different functions (e.g., photoreactivation by photo-lyases vs. clock/photoreceptor function of cryptochromes).

To understand the biochemical properties of mammalian cryptochromes, a panel of ten mutant mCRY1 expression constructs was generated, based on domains identified at the sequence level. We particularly paid attention to the most carboxy-terminal 100-120 amino acids of mCRY1, which most distinguishes the protein from mCRY2 as well as CRYs from other organisms (e.g., Drosophila) and which is lacking in photolyases. A schematic representation of the different mutant proteins, which include mutations in the nuclear localization signal (NLS) domains, deletion of the C-tail, deletion of the coiled coil, and mutation of tryptophan 320 to phenylalanine (in photolyase involved in intramolecular electron transport), is shown in Figure 2.

Using this panel of mutant constructs, we determined the domains involved in protein-protein interactions and subcellular localization of the protein and identified in the carboxyl terminus of mCRY1 the domain involved in association with mPER2 as well as with BMAL1 (Chaves et al. 2006; see Fig. 3). The interaction domain is represented by a coiled coil embedded in the carboxyl terminus, which is also present in Xenopus CRY (van der Schalie et al. 2007), but absent in Drosophila and plant CRYs. The carboxyl terminus was also shown to harbor a putative bipartite NLSc (Tamanini et al. 2005; Chaves et al. 2006). The PHR of mCRY1 contains a second NLS (NLSn), previously identified by Hirayama et al. (2003), which, however, is less potent than NLSc. Interestingly, combined deletion and/or mutagenesis of the NLS domains still allows the protein to reach the nucleus in an NLS-independent manner involving the coiled-coil domain. Previously, overexpressed mPER2 was shown to

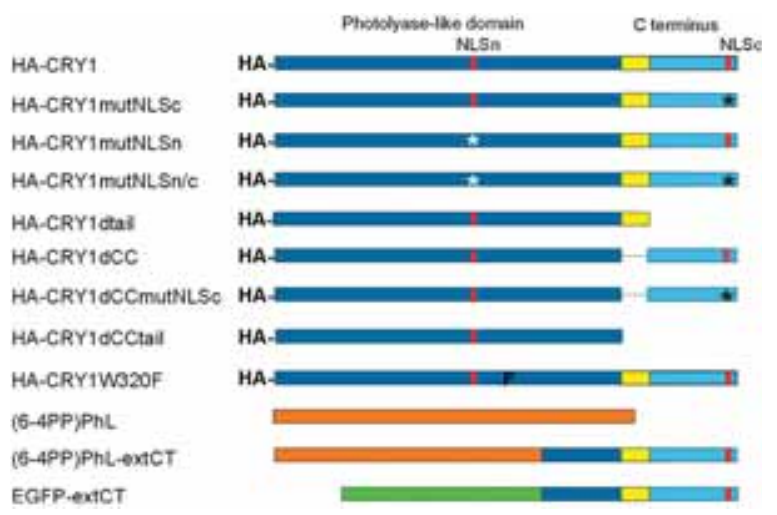

Figure 2. Schematic representation of (mutant) HA-CRY1 constructs, used for structure/function analysis of the mouse CRY1 protein. Mutations in the NLSc and NLSn are indicated by a star (black and white, respectively). (F) Mutation of tryptophan 320 to phenylalanine (W320F). (Dark blue) Photolyase-like PHR domain of mCRY1 (core domain); (light blue) carboxyl terminus (CT); (yellow) the coiled-coil domain.

shuttle between the nucleus and cytoplasm through the combined action of NLS and nuclear export sequences (NES) (Vielhaber et al. 2001; Yagita et al. 2002), whereas coexpression with mCRY1 (or mCRY2) caused completed nuclear localization of mPER2 (Kume et al. 1999). Following our observation that mPER2 and BMAL1 can competitively bind to the coiled coil of $\mathrm{MCRY} 1$ and facilitate nuclear localization of mCRY1mutNLSc, we pro-

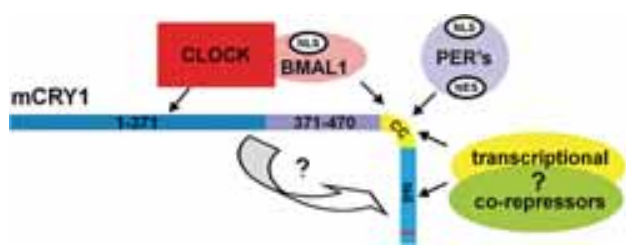

Figure 3. Model for the mechanism of action of mCRY1 within the mammalian core oscillator. The carboxyl terminus of mCRY1 is involved in association with MPER1 and mPER2 proteins and therefore regulates the stability and cellular localization of the latter proteins. The formation of the $\mathrm{mCRY} / \mathrm{mPER}$ complex occurs possibly through coiled-coil (CC) interactions, because a predicted coiled coil is also present in the mCRYbinding region of mPER proteins (data not shown). To achieve full nuclear localization, this protein complex requires both the mCRY1-NLSc and mPER2-NLS to counteract the NES-mediated nuclear export of mPER2. This molecular mechanism may explain the synchrony in nuclear localization of these proteins observed in vivo. Moreover, the photolyase-like core domain of mCRY1 interacts with CLOCK, whereas the coiled coil is involved in association with BMAL1. Our data also suggest competition between mPER2 and BMAL1 for binding with mCRY1, which supports the concept that the periodicity of the oscillator depends on temporal abundance and strength of interaction between the partners, as well as on the transcription inhibitory capacity of each complex. The inhibitory action of mCRY1 is likely achieved through an intermolecular interaction, which may impose a structural change in the carboxyl terminus, thereby allowing this domain to recruit transcriptional corepressor complexes. (Reprinted, with permission, from Chaves et al. 2006 [C ASM].) 


\section{STRUCTURE FUNCTION ANALYSIS OF MAMMALIAN CRYPTOCHROMES}

posed that the balance between the activities exerted by nuclear import and export signals harbored by CRY and PER2 determines the final subcellular localization of the complex at the steady-state level (Chaves et al. 2006).

Because the key function of $\mathrm{mCRY}$ proteins is to inhibit CLOCK/BMAL1-mediated transcription activation of Ebox genes in the negative limb of the mammalian circadian core oscillator, we also analyzed the performance of the various mutant mCRY1 proteins in in vitro CLOCK/ BMAL1 transcription assays. Although deletion of either the coiled coil or tail, or NLSc, did not affect the CLOCK/BMAL1 inhibitory capacity of MCRY1, complete removal of the carboxy-terminal extension (as in HA-CRY1dCCtail) rendered the protein inactive (Chaves et al. 2006). These data suggest that the "gain" of the unique carboxy-terminal extension during evolution has been critical in conferring core oscillator function to the PHR region. In line with this, although mutagenesis of conserved tryptophans (involved in intraprotein electron transport during photoactivation) abolishes photoreceptor function of Drosophila and butterfly CRY, it does not affect the CLOCK/BMAL1 transcription inhibitory capacity of mouse CRY1 (Zhu et al. 2005; Song et al. 2007).

Because the PHR of mCRY1 shares a high degree of homology with photolyase, it was therefore interesting to examine whether the carboxyl terminus of mCRY1 could convert a photolyase into a cryptochrome. Fusion of the carboxyl terminus of mCRY1 (amino acids 471-606) to Arabidopsis thaliana (6-4PP) photolyase did not result in a protein with CLOCK/BMAL1 inhibitory capacity. However, additional substitution of the last 100 amino acids of the photolyase PHR with that of the CRY1 PHR (amino acids 371-470) allowed the chimeric protein to inhibit CLOCK/BMAL1-driven transcription. When fused to enhanced green fluorescent protein (EGFP), the extended carboxyl terminus (amino acids 371-606) of mCRY1 was not able to inhibit CLOCK/BMAL1. This led us to suggest that the remainder of the photolyase/ cryptochrome core domain is very important for the clock function of cryptochrome proteins, likely through a complex network of interactions and intrinsic (PHR-like) structural requirements for proper transcription inhibition (Chaves et al. 2006). Indeed, it was shown that the carboxyl termini of animal and plant CRYs are intrinsically unstructured and that a stable tertiary structure is achieved via intramolecular interaction with the PHR (Ozgur and Sancar 2003). In a comparable series of experiments, van der Schalie et al. (2007) showed that Xenopus CRY photolyase fusion proteins maintain CLOCK/BMAL1 inhibitory capacity, further underlining the need for proper structural folding.

\section{FUTURE DIRECTIONS IN THE ANALYSIS OF MAMMALIAN CRYPTOCHROMES}

\section{Binding Partners of mCRY Proteins}

Deletion analysis has shown that the putative coiled-coil domain in the carboxyl terminus of mCRY1 is required for interacting with mPER1/2, BMAL1 (Chaves et al. 2006), and TIMELESS (F. Tamanini and G.T. van der Horst, in prep.). The carboxyl terminus further contains domains involved in nuclear import (Chaves et al. 2006) and, at least for mCRY2, phosphorylation (Sanada et al. 2004; Harada et al. 2005). It is likely that in addition to the already identified proteins, other factors are interacting with the carboxyl terminus, such as kinases and phosphatases, including perhaps FXBL3, which is the E3 ubiquitin ligase recently shown to target mCRY1 and mCRY2 to the degradation through the $26 \mathrm{~S}$ proteasome (Busino et al. 2007; Godinho et al. 2007; Siepka et al. 2007).

We are currently using amylose-bead-immobilized purified MBP-CRY1 and MBP-CRY2 carboxyl terminal domains to probe mouse tissue (e.g., liver) lysates to identify tail-binding proteins using MALDI-TOF, as well as more sophisticated methods (MALDI-TOF-TOF or ESIQ-TOF) when necessary. We believe that the identification of protein partners will not only further elucidate the function of CRY proteins in the mammalian clock, but may also disclose new functions of these proteins.

\section{Opposite Phenotypes of mCry $1^{-/-}$ and $\mathrm{mCry}^{-{ }^{--}}$Mice}

Analysis of the phenotype of $m C r y$ mutant mice revealed that the $m C r y l$ gene drives long-period circadian clockworks, whereas the $m C r y 2$ gene confers short periodicity to the circadian oscillator (Okamura et al. 1999; van der Horst et al. 1999). Although it is possible that the two genes/proteins could be expressed in different circadian time windows, CRY1 and CRY2 proteins reach the nuclear compartment in a synchronous manner (Field et al. 2000; Lee et al. 2001). We therefore favor the idea that the two proteins have opposing circadian effects because of sequence differences translating in different activities in and/or posttranslational regulation of the clock. Vanselow et al. (2006) launched the interesting hypothesis that shortand long-period phenotypes of $\mathrm{Cry} \mathrm{I}^{-/-}$and $\mathrm{Cry} 2^{-/-}$mutant mice could be the result of alterations in the phosphorylation patterns of PER proteins. The lack of CRY1 or CRY2 would differentially influence the phosphorylation state of PER proteins and thus could modulate the circadian period in opposite directions. In line with this idea, phosphorylation of mPER2 at different serines can have opposite effects on clock speed: Whereas phosphorylation of PER2 at the FASPS position and immediate downstream positions causes PER2 protein stabilization, phosphorylation by CKI $/ \delta$ at other sites leads to mPER2 protein degradation (Toh et al. 2001; Xu et al. 2007). In this scenario, the PER proteins are primarily responsible for proper timing of the mammalian circadian oscillator. To test this hypothesis, a dynamic and quantitative phosphorylation site mapping of PER proteins in $\mathrm{Cry} \mathrm{I}^{-/-}$and $\mathrm{Cry} 2^{-/-}$mouse cells or tissues would be needed.

\section{Transcription Repression}

Inhibition of the CLOCK-BMAL1 heterodimer is independent of light and is triggered at relatively low doses of CRY. Although the mechanism underlying CLOCK/ BMAL1 repression is not known, some ideas have been proposed. One hypothesis is that $\mathrm{mCRY}$ proteins repress 
CLOCK-BMAL1 by reducing CLOCK-BMAL1's affinity for the E box. Another idea is that CRYs inhibit CLOCK-BMAL1 by interacting with the heterodimer and then recruiting histone deacetylases or inhibiting histone acetylases (Etchegaray et al. 2003). Surely, an area of exploration would be the study of the impact of mCRYs on the posttranslational properties of CLOCK and BMAL1 (Dardente et al. 2007).

\section{PHR Dimerization, a Key to Understanding the Evolution of the Mammalian Clock?}

Recently, an illuminating report showed that homodimerization of the PHR of AtCRY1 was mandatory for subsequent light activation of its carboxyl terminus (CCT1) (Sang et al. 2005). The activated CCT1 would then bind and inhibit the E3 ubiquitin ligase COP1, thereby allowing the accumulation of a set of transcription factors (e.g., HY5) that initiate the photomorphogenic program (Wang et al. 2001; Yang et al. 2001). Thus far, all of our efforts to detect a functional homodimerization of mCRY proteins have failed (F. Tamanini and G.T. van der Horst, unpubl.). This lack of positive results is somewhat surprising because comparison of the PHR among members of the cryptochrome/photolyase family, as well as molecular modeling of the three-dimensional structures of the PHR, suggests an almost identical folding of the PHR in all cryptochromes. Moreover, interaction between the PHR of AtCRY1 and mCRY2 with their corresponding carboxyl termini has been shown to be an important event in establishing the stable tertiary structure of these proteins, suggesting a common biochemical mode of action for cryptochromes (Partch et al. 2005). Then why does mammalian mCRY1 not homodimerize as does its plant homolog? Given the difference in function, it is tempting to speculate that the necessity for homodimerization or heterodimerization is restricted to photoreceptor function. In mammals, mCRYs have a dominant role in the transcriptional-posttranslational feedback loops governing circadian rhythms in the SCN and peripheral tissues. With the exception of the SCN, which responds to light through neuronal signaling via the retinohypothalamic tract, the mammalian circadian oscillator does not respond to light. Therefore, one possibility is that the lack of homodimerization and heterodimerization of mCRY proteins underlines the absence of any photoreceptor function in this protein (although we cannot rule out light responsiveness of the mCRY PHR in the retina). If this interpretation is correct, the prediction would be that Drosophila CRY (Stanewsky 2002), which acts as both a circadian photoreceptor (like AtCRY) and a central clock component (like mCRYs), might have preserved the capacity to homodimerize as a regulatory mechanism for light signaling.

\section{ACKNOWLEDGMENTS}

We thank Dr. Andre Eker for stimulating discussion. This work was supported in part by grants from the Netherlands Organization for Scientific Research (ZonMW Vici 918.36.619 and NWO-CW 700.51.304), SenterNovem
(BSIK03053), and the European Community (BrainTime QLG3-CT-2002-01829; EUCLOCK LSHG-CT-2006018741) to G.T.J.vdH.

\section{REFERENCES}

Aubert C., Vos M.H., Mathis P., Eker A.P., and Brettel K. 2000 Intraprotein radical transfer during photoactivation of DNA photolyase. Nature 405: 586.

Bohr V.A., Smith C.A., Okumoto D.S., and Hanawalt P.C. 1985. DNA repair in an active gene: Removal of pyrimidine dimers from the DHFR gene of CHO cells is much more efficient than in the genome overall. Cell 40: 359 .

Bouly J.P., Schleicher E., Dionisio-Sese M., Vandenbussche F., Van Der Straeten D., Bakrim N., Meier S., Batschauer A., Galland P., Bittl R., and Ahmad M. 2007. Cryptochrome blue light photoreceptors are activated through interconversion of flavin redox states. J. Biol. Chem. 282: 9383.

Busino L., Bassermann F., Maiolica A., Lee C., Nolan P.M., Godinho S.I., Draetta G.F., and Pagano M. 2007. SCFFbxl3 controls the oscillation of the circadian clock by directing the degradation of cryptochrome proteins. Science 316: 900.

Busza A., Emery-Le M., Rosbash M., and Emery P. 2004. Roles of the two Drosophila CRYPTOCHROME structural domains in circadian photoreception. Science 304: 1503.

Carell T., Burgdorf L.T., Kundu L.M., and Cichon M. 2001. The mechanism of action of DNA photolyases. Curr. Opin. Chem. Biol. 5: 491.

Cashmore A.R. 2003. Cryptochromes: Enabling plants and animals to determine circadian time. Cell 114: 537.

Cashmore A.R., Jarillo J.A., Wu Y.J., and Liu D. 1999. Cryptochromes: Blue light receptors for plants and animals. Science 284: 760.

Chaves I., Yagita K., Barnhoorn S., Okamura H., van der Horst G.T., and Tamanini F. 2006. Functional evolution of the photolyase/cryptochrome protein family: Importance of the $\mathrm{C}$ terminus of mammalian CRY1 for circadian core oscillator performance. Mol. Cell. Biol. 26: 1743.

Dardente H., Fortier E.E., Martineau V., and Cermakian N. 2007. Cryptochromes impair phosphorylation of transcriptional activators in the clock: A general mechanism for circadian repression. Biochem. J. 402: 525 .

de Laat W.L., Jaspers N.G., and Hoeijmakers J.H. 1999. Molecular mechanism of nucleotide excision repair. Genes Dev. 13: 768.

Emery P., So W.V., Kaneko M., Hall J.C., and Rosbash M. 1998. CRY, a Drosophila clock and light-regulated cryptochrome, is a major contributor to circadian rhythm resetting and photosensitivity. Cell 95: 669

Etchegaray J.P., Lee C., Wade P.A., and Reppert S.M. 2003. Rhythmic histone acetylation underlies transcription in the mammalian circadian clock. Nature 421: 177.

Field M.D., Maywood E.S., O’Brien J.A., Weaver D.R., Reppert S.M., and Hastings M.H. 2000. Analysis of clock proteins in mouse SCN demonstrates phylogenetic divergence of the circadian clockwork and resetting mechanisms. Neuron 25: 437.

Friedberg E.C. 1996. Relationships between DNA repair and transcription. Annu. Rev. Biochem. 65: 15.

Garinis G.A., Mitchell J.R., Moorhouse M.J., Hanada K., de Waard H., Vandeputte D., Jans J., Brand K., Smid M., van der Spek P.J., Hoeijmakers J.H., Kanaar R., and van der Horst G.T. 2005. Transcriptome analysis reveals cyclobutane pyrimidine dimers as a major source of UV-induced DNA breaks. EMBO J. 24: 3952.

Godinho S.I., Maywood E.S., Shaw L., Tucci V., Barnard A.R., Busino L., Pagano M., Kendall R., Quwailid M.M., Romero M.R., O’Neill J., Chesham J.E., Brooker D., Lalanne Z., Hastings M.H., and Nolan P.M. 2007. The after-hours mutant reveals a role for Fbx13 in determining mammalian circadian period. Science 316: 897.

Griffin E.A., Jr., Staknis D., and Weitz C.J. 1999. Light-independent role of CRY1 and CRY2 in the mammalian circadian clock. Science 286: 768. 


\section{STRUCTURE FUNCTION ANALYSIS OF MAMMALIAN CRYPTOCHROMES}

Harada Y., Sakai M., Kurabayashi N., Hirota T., and Fukada Y. 2005. Ser-557-phosphorylated mCRY2 is degraded upon synergistic phosphorylation by glycogen synthase kinase- $3 \beta$. $J$. Biol. Chem. 280: 31714.

Hirayama J., Nakamura H., Ishikawa T., Kobayashi Y., and Todo T. 2003. Functional and structural analyses of cryptochrome. Vertebrate CRY regions responsible for interaction with the CLOCK:BMAL1 heterodimer and its nuclear localization. $J$. Biol. Chem. 278: 35620.

Honma S., Kawamoto T., Takagi Y., Fujimoto K., Sato F., Noshiro M., Kato Y., and Honma K. 2002. Dec1 and Dec2 are regulators of the mammalian molecular clock. Nature 419: 841 .

Jans J., Schul W., Sert Y.G., Rijksen Y., Rebel H., Eker A.P., Nakajima S., van Steeg H., de Gruijl F.R., Yasui A., Hoeijmakers J.H., and van der Horst G.T. 2005. Powerful skin cancer protection by a CPD-photolyase transgene. Curr. Biol. 15: 105 .

Jans J., Garinis G.A., Schul W., van Oudenaren A., Moorhouse M., Smid M., Sert Y.G., van der Velde A., Rijksen Y., de Gruijl F.R., van der Spek P.J., Yasui A., Hoeijmakers J.H., Leenen P.J., and van der Horst G.T. 2006. Differential role of basal keratinocytes in UV-induced immunosuppression and skin cancer. Mol. Cell. Biol. 26: 8515 .

Kume K., Zylka M.J., Sriram S., Shearman L.P., Weaver D.R., Jin X., Maywood E.S., Hastings M.H., and Reppert S.M. 1999. mCRY1 and mCRY2 are essential components of the negative limb of the circadian clock feedback loop. Cell 98: 193.

Lee C., Etchegaray J.P., Cagampang F.R., Loudon A.S., and Reppert S.M. 2001. Posttranslational mechanisms regulate the mammalian circadian clock. Cell 107: 855.

Mees A., Klar T., Gnau P., Hennecke U., Eker A.P., Carell T., and Essen L.O. 2004. Crystal structure of a photolyase bound to a CPD-like DNA lesion after in situ repair. Science 306: 1789.

Okamura H., Miyake S., Sumi Y., Yamaguchi S., Yasui A., Muijtjens M., Hoeijmakers J.H., and van der Horst G.T. 1999. Photic induction of mPer1 and mPer2 in cry-deficient mice lacking a biological clock. Science 286: 2531.

Ozgur S. and Sancar A. 2003. Purification and properties of human blue-light photoreceptor cryptochrome 2. Biochemistry 42: 2926.

Partch C.L., Clarkson M.W., Ozgur S., Lee A.L., and Sancar A. 2005. Role of structural plasticity in signal transduction by the cryptochrome blue-light photoreceptor. Biochemistry 44: 3795.

Ralph M.R., Foster R.G., Davis F.C., and Menaker M. 1990. Transplanted suprachiasmatic nucleus determines circadian period. Science 247: 975.

Reppert S.M. and Weaver D.R. 2002. Coordination of circadian timing in mammals. Nature 418: 935.

Ripperger J.A., Shearman L.P., Reppert S.M., and Schibler U. 2000. CLOCK, an essential pacemaker component, controls expression of the circadian transcription factor DBP. Genes Dev. 14: 679 .

Sanada K., Harada Y., Sakai M., Todo T., and Fukada Y. 2004. Serine phosphorylation of mCRY1 and mCRY2 by mitogenactivated protein kinase. Genes Cells 9: 697.

Sancar A. 2003. Structure and function of DNA photolyase and cryptochrome blue-light photoreceptors. Chem. Rev. 103: 2203.

Sang Y., Li Q.H., Rubio V., Zhang Y.C., Mao J., Deng X.W., and Yang H.Q. 2005. N-terminal domain-mediated homodimerization is required for photoreceptor activity of Arabidopsis CRYPTOCHROME 1. Plant Cell 17: 1569.

Schul W., Jans J., Rijksen Y.M., Klemann K.H., Eker A.P., de Wit J., Nikaido O., Nakajima S., Yasui A., Hoeijmakers J.H., and van der Horst G.T. 2002. Enhanced repair of cyclobutane pyrimidine dimers and improved UV resistance in photolyase transgenic mice. $E M B O$ J. 21: 4719.

Shearman L.P., Sriram S., Weaver D.R., Maywood E.S., Chaves I., Zheng B., Kume K., Lee C.C., van der Horst G.T., Hastings M.H., and Reppert S.M. 2000. Interacting molecular loops in the mammalian circadian clock. Science 288: 1013.

Siepka S.M., Yoo S.H., Park J., Song W., Kumar V., Hu Y., Lee C., and Takahashi J.S. 2007. Circadian mutant Overtime reveals F-box protein FBXL3 regulation of cryptochrome and period gene expression. Cell 129: 1011

Song S.H., Oztürk N., Denaro T.R., Arat N.O., Kao Y.T., Zhu H., Zhong D., Reppert S.M., and Sancar A. 2007. Formation and function of flavin anion radical in cryptochrome 1 blue-light photoreceptor of monarch butterfly. J. Biol. Chem. 282: 17608.

Stanewsky R. 2002. Clock mechanisms in Drosophila. Cell Tissue Res. 309: 11.

Stanewsky R., Kaneko M., Emery P., Beretta B., Wager-Smith K., Kay S.A., Rosbash M., and Hall J.C. 1998. The $c r y^{b}$ mutation identifies cryptochrome as a circadian photoreceptor in Drosophila. Cell 95: 681.

Suarez-Lopez P. and Coupland G. 1998. Plants see the blue light. Science 279: 1323.

Tamada T., Kitadokoro K., Higuchi Y., Inaka K., Yasui A., de Ruiter P.E., Eker A.P., and Miki K. 1997. Crystal structure of DNA photolyase from Anacystis nidulans. Nat. Struct. Biol. 4: 887.

Tamanini F., Yagita K., Okamura H., and van der Horst G.T. 2005. Nucleocytoplasmic shuttling of clock proteins. Methods Enzymol. 393: 418.

Todo T., Tsuji H., Otoshi E., Hitomi K., Kim S.T., and Ikenaga M. 1997. Characterization of a human homolog of (6-4) photolyase. Mutat. Res. 384: 195.

Toh K.L., Jones C.R., He Y., Eide E.J., Hinz W.A., Virshup D.M., Ptacek L.J., and Fu Y.H. 2001. An hPer2 phosphorylation site mutation in familial advanced sleep phase syndrome. Science 291: 1040.

van der Horst G.T., Muijtjens M., Kobayashi K., Takano R., Kanno S., Takao M., de Wit J., Verkerk A., Eker A.P., van Leenen D., Buijs R., Bootsma D., Hoeijmakers J.H., and Yasui A. 1999. Mammalian Cry1 and Cry2 are essential for maintenance of circadian rhythms. Nature 398: 627.

van der Schalie E.A., Conte F.E., Marz K.E., and Green C.B. 2007. Structure/function analysis of Xenopus cryptochromes 1 and 2 reveals differential nuclear localization mechanisms and functional domains important for interaction with and repression of CLOCK-BMAL1. Mol. Cell. Biol. 27: 2120.

van der Spek P.J., Kobayashi K., Bootsma D., Takao M., Eker A.P., and Yasui A. 1996. Cloning, tissue expression, and mapping of a human photolyase homolog with similarity to plant blue-light receptors. Genomics 37: 177.

Vanselow K., Vanselow J.T., Westermark P.O., Reischl S., Maier B., Korte T., Herrmann A., Herzel H., Schlosser A., and Kramer A. 2006. Differential effects of PER2 phosphorylation: Molecular basis for the human familial advanced sleep phase syndrome (FASPS). Genes Dev. 20: 2660.

Vielhaber E.L., Duricka D., Ullman K.S., and Virshup D.M. 2001. Nuclear export of mammalian PERIOD proteins. J. Biol. Chem. 276: 45921 .

Vitaterna M.H., Selby C.P., Todo T., Niwa H., Thompson C., Fruechte E.M., Hitomi K., Thresher R.J., Ishikawa T., Miyazaki J., Takahashi J.S., and Sancar S. 1999. Differential regulation of mammalian period genes and circadian rhythmicity by cryptochromes 1 and 2. Proc. Natl. Acad. Sci. 96: 12114.

Wang H., Ma L.G., Li J.M., Zhao H.Y., and Deng X.W. 2001. Direct interaction of Arabidopsis cryptochromes with COP1 in light control development. Science 294: 154.

Xu Y., Toh K.L., Jones C.R., Shin J.Y., Fu Y.H., and Ptacek L.J. 2007. Modeling of a human circadian mutation yields insights into clock regulation by PER2. Cell 128: 59 .

Yagita K., Tamanini F., Yasuda M., Hoeijmakers J.H., van der Horst G.T., and Okamura H. 2002. Nucleocytoplasmic shuttling and mCRY-dependent inhibition of ubiquitylation of the mPER2 clock protein. EMBO J. 21: 1301.

Yang H.Q., Tang R.H., and Cashmore A.R. 2001. The signaling mechanism of Arabidopsis CRY1 involves direct interaction with COP1. Plant Cell 13: 2573.

Yang H.Q., Wu Y.J., Tang R.H., Liu D., Liu Y., and Cashmore A.R. 2000. The $\mathrm{C}$ termini of Arabidopsis cryptochromes mediate a constitutive light response. Cell 103: 815.

Zhu H., Yuan Q., Briscoe A.D., Froy O., Casselman A., and Reppert S.M. 2005. The two CRYs of the butterfly. Curr. Biol. 15: R953. 


\section{$8_{\text {SYMPOS }}^{\infty}$ Cold Spring Harbor Symposia SYMPOSIA on Quantitative Biology}

\section{Structure Function Analysis of Mammalian Cryptochromes}

F. Tamanini, I. Chaves, M. I. Bajek, et al.

Cold Spring Harb Symp Quant Biol 2007 72: 133-139

Access the most recent version at doi:10.1101/sqb.2007.72.066

References This article cites 58 articles, 29 of which can be accessed free at: http://symposium.cshlp.org/content/72/133.full.html\#ref-list-1

\section{License} Email Alerting $\begin{aligned} & \text { Receive free email alerts when new articles cite this article - sign up in the box at the } \\ & \text { Service }\end{aligned}$ top right corner of the article or click here. 